\title{
High-Contrast Imaging and High-Resolution Spectroscopy Observation of Exoplanets
}

\author{
Ji Wang ${ }^{\mathrm{a}}$, Dimitri Mawet ${ }^{\mathrm{a}}$, Renyu Hu${ }^{\mathrm{b}, \mathrm{c}}$, and Björn Benneke ${ }^{\mathrm{c}}$ \\ a Department of Astronomy, California Institute of Technology, Pasadena, CA 91125, USA \\ ${ }^{\mathrm{b} J e t}$ Propulsion Laboratory, California Institute of Technology, Pasadena, CA 91109, USA \\ ${ }^{c}$ Division of Geological and Planetary Sciences, California Institute of Technology, Pasadena, \\ CA 91125, USA
}

\begin{abstract}
Detection and characterization of exoplanets faces challenges of smaller angular separation and high contrast between exoplanets and their host stars. High contrast imaging (HCI) instruments equipped with coronagraphs are built to meet these challenges, providing a way of spatially suppressing and separating stellar flux from that of a planet. Another way of separating stellar flux can be achieved by high-resolution spectroscopy (HRS), exploiting the fact that spectral features are different between a star and a planet. Observing exoplanets with HCI+HRS will achieve a higher contrast than the spatial or the spectroscopic method alone, improving the sensitivity to planet detection and enabling the study of the physical and chemical processes. Here, we simulate the performance of a HCI+HRS instrument (i.e., the upgrade Keck NIRSPEC and the fiber injection unit) to study its potential in detecting and characterizing currently known directly imaged planets. The simulation considers the spectral information content of an exoplanet, telescope and instrument specifications and realistic noise sources. The result of the simulation helps to set system requirement and informs designs at system-level.
\end{abstract}

Keywords: Exoplanet, coronagraph, high-contrast imaging, high-resolution spectrograph, atmospheric modeling

\section{INTRODUCTION}

Thousands of exoplanets have been discovered to date. Detailed characterization of exoplanets is the logical next step. Directly-imaged exoplanets are excellent targets for chemical composition study [1-4]. However, direct imaging and characterization of exoplanets is challenging because of the small angular separation and high contrast between exoplanets and their host stars. Both constraints can be mitigated by a high-contrast imaging (HCI) system consisting of an extreme adaptive optics (AO) system and a coronagraphic system. Recently, Snellen et al. $(2015)^{5}$ suggested that contrast can be further improved by coupling a high-resolution spectrograph (HRS) with a HCI system. In this HCI+HRS scheme, the HCI system serves as a spatial filter to separate the light from the star and the planet; HRS serves as spectral filter because of different spectral features of the stellar spectrum and the planetary spectrum, e.g., different absorption lines and different radial velocities.

Using HRS as a way of spectral filtering has been successfully demonstrated for a number of studies. For transiting planets, high-resolution transmission spectroscopy has been used to detect molecular gas [6-8]. At a high spectral resolution, resolved molecular lines can be used to study day-to-night-side wind velocity [6], providing an ultimate test for 3D exoplanet atmosphere models [9]. For radial velocity detected planets, spectral lines of a planet can be separated from stellar lines due to drastically different radial velocities $\left(\gtrsim 50 \mathrm{~km} \mathrm{~s}^{-1}\right)$. The radial velocity of a planet can thus be measured to break the degeneracy between the true planet mass and orbital inclination [10-13]. Moreover, high-resolution spectroscopy permits a detailed study of spectral lines arising from a planet atmosphere. This approach led to the first measurement of planet rotation velocity [14]. With time-series high-resolution spectroscopy, surface features such as cloud or spot coverage can be inferred

Further author information: (Send correspondence to Ji Wang)

E-mail: ji.wang@caltech.edu, Telephone: 16263954981

Modeling, Systems Engineering, and Project Management for Astronomy VII, edited by George Z. Angeli, Philippe Dierickx, Proc. of SPIE Vol. 9911, 99112T

(c) 2016 SPIE $\cdot$ CCC code: $0277-786 X / 16 / \$ 18 \cdot$ doi: $10.1117 / 12.2235216$

Proc. of SPIE Vol. $991199112 \mathrm{~T}-1$ 
via the Doppler imaging technique. This technique has been demonstrated on the closest brown dwarf system, Luhman 16 AB [15].

Combining HCI and HRS, ground-based HCI-HRS instruments can deliver a high contrast level and thus provide an excellent resource for follow-up observation for directly imaged planets. Here, we provide a pipeline to simulate HCI+HRS observation. This pipeline considers astrophysical processes and instrumental effects to produce a realistic simulation. Result of the simulation can be used to set system requirements and shed light on designs at system-level. We apply the pipeline to a case that concerns ground-based observation of HR 8799 planetary system with the upgraded Keck NIRSPEC and a fiber injection unit (FIU) that connects the coronagraphic system with the spectroscopic system.

\section{DESCRIPTION OF SIMULATION}

We describe the procedures to simulate the end-to-end performance of a HCI-HRS system, from the absorption and thermal emission spectrum of a planet to the reduced spectrum and the subsequent data analyses. Fig. 1 provides the flow chart and the system-related inputs of the simulation. The resulting data products and their quality, e.g., signal to noise ratio (SNR), will shed light on the system requirements, e.g., coronagraph design and the performance of the $\mathrm{AO}$ system.

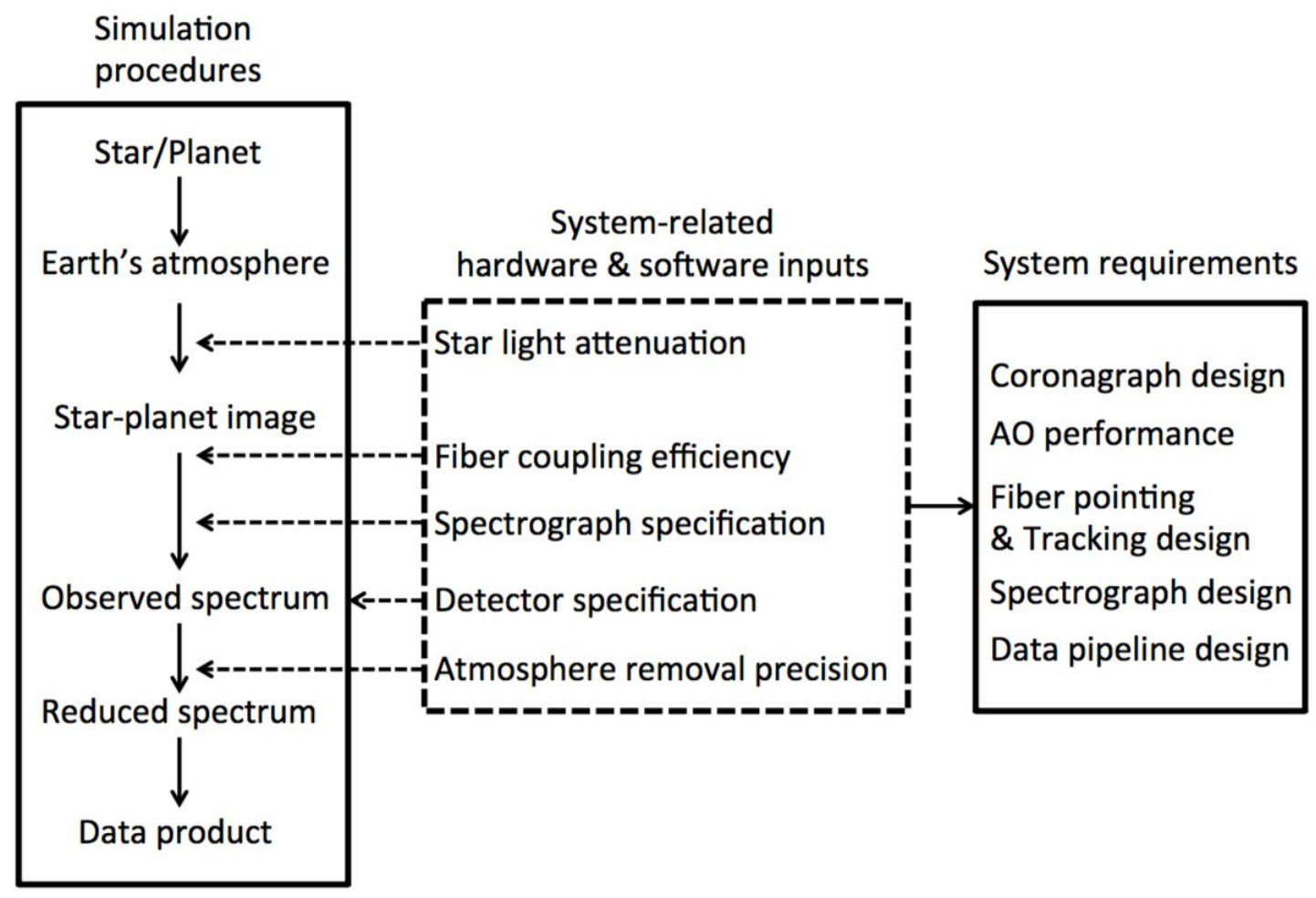

Figure 1. Flow chart for simulation procedures and system-related inputs at certain steps as marked by dashed arrows. Result of the simulation can be used to set system requirements and inform design at system-level.

The simulation starts with spectra of a star and its planet, which are obtained from multiple sources: PHOENIX BT-Settl grids [16] for integrated spectra for gas giant planets, SCARLET for molecular-by-molecular spectra for gas giant planets [17], and photochemistry-thermochemistry modeled atmospheres for low-mass planets [18-21]. The photon flux of a star or a planet scales with distance, telescope aperture, instrument throughput and exposure time. In addition, photons are absorbed and re-emitted by the Earth's atmosphere. We use the atmospheric absorption and emission spectra from Mauna Kea mountain in Hawaii [22]. At the telescope image plane after $\mathrm{AO}$ and coronagraphic system, star light is attenuated whereas planet light is mostly intact. We use 
the term star light attenuation level to quantify the attenuation factor, i.e., the amount of stellar light entering spectrograph divided by the total amount of stellar light entering the telescope. The spectrograph is linked to the $\mathrm{AO}$ and coronagraphic system by a single mode fiber, which is positioned at the planet location. Both the planet light and the attenuated star light enters the spectrograph and is dispersed with a certain spectral resolution. The dispersed light is then recorded on a detector for which we consider its readout noise and dark current in error budget. Equation 1 describes the flux recorded on detector, in which $f$ denotes spectrum, i.e., flux as a function of wavelength, and subscripts denotes sources of spectra, $\mathrm{C}$ is the star light attenuation level. Detector noise sources include readout noise $(\mathrm{RN})$ and dark current (dark). The observed spectrum is then reduced assuming that certain fraction of atmospheric absorption and emission is removed, which results in a reduced spectrum. Equation 2 describes the reduced spectrum, where $\sigma$ is the removal level of the Earth atmosphere and $\delta$ is the noise based on Equation 1, i.e., the square root of the quadrature summation of photon noise and detector noise. The reduced spectrum will be further analyzed and turned into data products.

$$
\begin{gathered}
\mathrm{f}_{\text {detector }}=\left(\mathrm{f}_{\text {planet }}+\mathrm{f}_{\text {star }} \times \mathrm{C}\right) \times \mathrm{f}_{\text {transmission }}+\mathrm{f}_{\text {sky }}+\mathrm{RN}+\text { dark } \times \mathrm{t}_{\text {exp }}, \\
\mathrm{f}_{\text {reduced }}=\left(\mathrm{f}_{\text {planet }}+\mathrm{f}_{\text {star }} \times \mathrm{C}\right) \times\left(1-\sigma \times\left(1-\mathrm{f}_{\text {transmission }}\right)\right)+\sigma \times \mathrm{f}_{\text {sky }}+\delta,
\end{gathered}
$$

The data products include cross correlation function (CCF) and planet surface brightness map. The CCF is obtained by cross correlating the reduced spectrum with a synthetic planet spectrum. The cross-correlation masks out regions that are heavily contaminated by sky emission lines, i.e., wavelengths with sky emission levels higher than half of flux fluctuation. CCF SNR is defined as the ratio between the CCF peak and the RMS of $\mathrm{CCF}$ outside the peak region $(\Delta V>15,000 \mathrm{~km} / \mathrm{s})$. We also ensure that the peak of the CCF is consistent with the radial velocity of the planetary system. This is because that CCF peak could be due to the residual of the Earth's atmosphere for certain species that also exist in the Earth's atmosphere. Rotation of a planet causes modulation of surface brightness, which results in periodical variation spectral lines. By analyzing the time-resolved line variation, surface brightness map can be retrieved using the Doppler Imaging technique [15]. We obtain surface brightness map by generating time-resolved reduced spectrum and analyzing them with the maximum entropy method $[23,24]$. We then use CCF SNR and the quality of retrieved surface brightness map to inform system design and requirement.

\section{SIMULATING GROUND-BASED HCI+HRS OBSERVATION OF HR 8799 E}

\subsection{HR 8799 e}

Table 1. Inputs For HR 8799 e Simulation

\begin{tabular}{|l|l|l|l|l|l|}
\hline Telescope/Instrument & Values & \multicolumn{2}{|c|}{ Star } & \multicolumn{2}{c|}{ Planet } \\
\hline Telescope Aperture & $10 \mathrm{~m}$ & $\mathrm{~T}_{\text {eff }}$ & $7193 \mathrm{~K}$ & $\mathrm{~T}_{\text {eff }}$ & $1200 \mathrm{~K}$ \\
\hline End-to-End Throughput & $10 \%$ & $\log (\mathrm{g})$ & 4.0 & $\log (\mathrm{g})$ & 3.7 \\
\hline Spectral Resolution & 37500 & V sin $i$ & $37.5 \mathrm{~km} / \mathrm{s}$ & $\mathrm{V} \sin i$ & $15.0 \mathrm{~km} / \mathrm{s}$ \\
\hline Exposure Time & $3600 \mathrm{~s}$ & Orbital Inclination & $<40 \mathrm{deg}$ & Orbital Inclination & $28 \mathrm{deg}$ \\
\hline Readout Noise & $3.0 \mathrm{e}^{-1}$ & Radial Velocity & $-12.6 \mathrm{~km} / \mathrm{s}$ & Metallicity & $0.5 \mathrm{dex}$ \\
\hline Dark Current & $0.01 \mathrm{e}^{-1} / \mathrm{s}$ & Distance & $39.4 \mathrm{pc}$ & Semi-major Axis & $17 \mathrm{AU}$ \\
\hline
\end{tabular}

Planet $\mathrm{e}$ is the most challenging planet to observe among all 4 planets in HR 8799 system because of its proximity to the host star. Following the methods detailed in the previous section, we simulate observations with a HCI+HRS instrument (FIU plus upgrade NIRSPEC) at Keck. Input parameters for planet, star, telescope and instrument are provided in Table 1. Because spectra for exact effective temperature and surface gravity are not available from the BT-Settle models, we use the spectrum with $T_{\text {eff }}=1200 \mathrm{~K}$ and $\log (\mathrm{g})=3.5$ as the 
input planet spectrum. We use the spectrum with $T_{\text {eff }}=7400 \mathrm{~K}$ and $\log (\mathrm{g})=4.0$ as the input star spectrum. Metallicity $[\mathrm{Fe} / \mathrm{H}]$ is set to zero for both planet and star. We adjust planet and star flux so that the model flux and the absolute flux measured from photometry is consistent within uncertainty (Fig. 2). We adopt values from [25] for absolute flux measurement. For template spectrum that is used for cross correlation for planet detection, we use (1) the same spectrum as the input planet spectrum or (2) the combined single-molecular spectrum of $\mathrm{CO}, \mathrm{CH}_{4}$, and $\mathrm{H}_{2} \mathrm{O}$. In the first case, we assume an ideal case in which we have a perfect knowledge of planet spectrum. In the second case, we have limited information of the intrinsic planet spectrum.

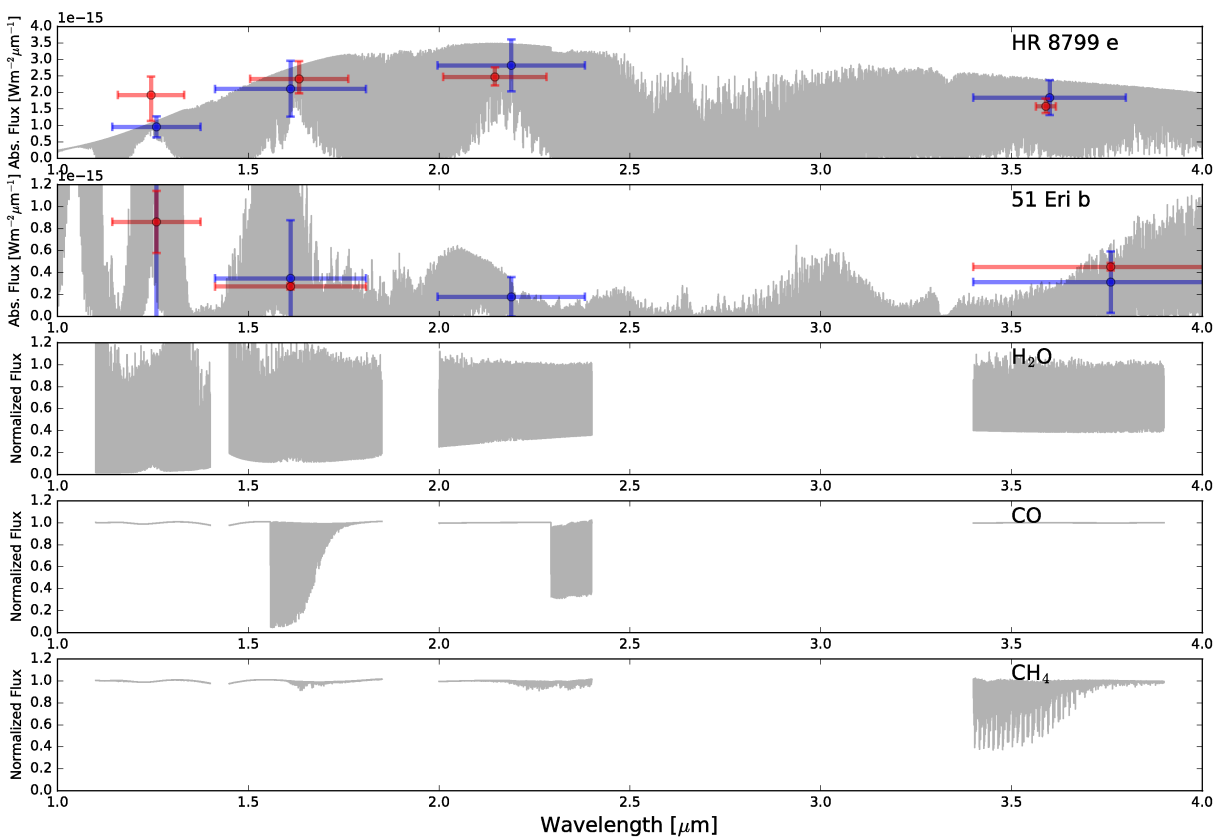

Figure 2. Top two panels: BT-Settle spectra from HR 8799 e and 51 Eri b and comparison of absolute flux between model and observation [25] in different photometric bands. Bottom three panels: normalized spectra for individual molecular species. These spectra are used for detection of molecular species in the atmosphere of HR 8799 e and 51 Eri b.

\subsubsection{Limiting Factors for CCF SNR}

We consider four cases in CCF SNR calculation (Fig. 3). In case one, CCF SNR is limited by the intrinsic fluctuation in regions where we calculate noise level, i.e., $\Delta \mathrm{V}>15,000 \mathrm{~km} \cdot \mathrm{s}^{-1}$. In theory, one can remove the intrinsic CCF fluctuation by subtracting the noiseless CCF from the noisy CCF. The remaining noise level is due to photon noise. The photon noise limited case is the second case we consider. It is also the case in which CCF SNR is the highest because there is no systematics error sources. The limiting photon noise can be from various sources. At low level of star light attenuation, the dominating noise source is always the photon noise from the star. At higher level of star light attenuation, the limiting photon noise can be sky background emission (e..g, $L$ band) or the planet itself (e.g., $J, H, K$ band). The photon noise limited case is the most optimistic case in which we have perfect knowledge of the planet and the star and the result is not limited by systematics.

In practice, however, we do not know the noiseless planet and star spectra a priori, so we do not know the noiseless CCF. Therefore, CCF SNR is certainly limited by systematics. In addition to the intrinsic CCF fluctuation case, we also consider two other cases in which systematics dominates the CCF SNR. We calculate the spectral line spread function (LSF) of the reduced planet spectrum using the least square deconvolution (LSD) technique [26]. We compare the peak of LSF and the fluctuation level in regions that are 5 resolution 
elements away from the LSF center. We define LSD SNR as the ratio between the peak and the fluctuation and plot LSD SNR in Fig. 3. This case delivers comparable or slightly better results than the CCF fluctuation case, especially at high SNR regime [26].

The last case we consider is a mismatch between the intrinsic and the modeled planet spectrum. For the intrinsic planet spectrum, we use a BT-Settle spectrum with $T_{\text {eff }}=1200 \mathrm{~K}$ and $\log (\mathrm{g})=3.5$. For the modeled planet spectrum, we combined the single-molecular spectra for $\mathrm{CO}, \mathrm{CH}_{4}$, and $\mathrm{H}_{2} \mathrm{O}$. This case gives the lowest $\mathrm{CCF}$ SNR because of the mismatched spectrum and the limiting systematics. This a pessimistic case but highlights the importance of planet spectrum modeling and a good understanding of systematics in characterizing exoplanet with the cross correlation method.
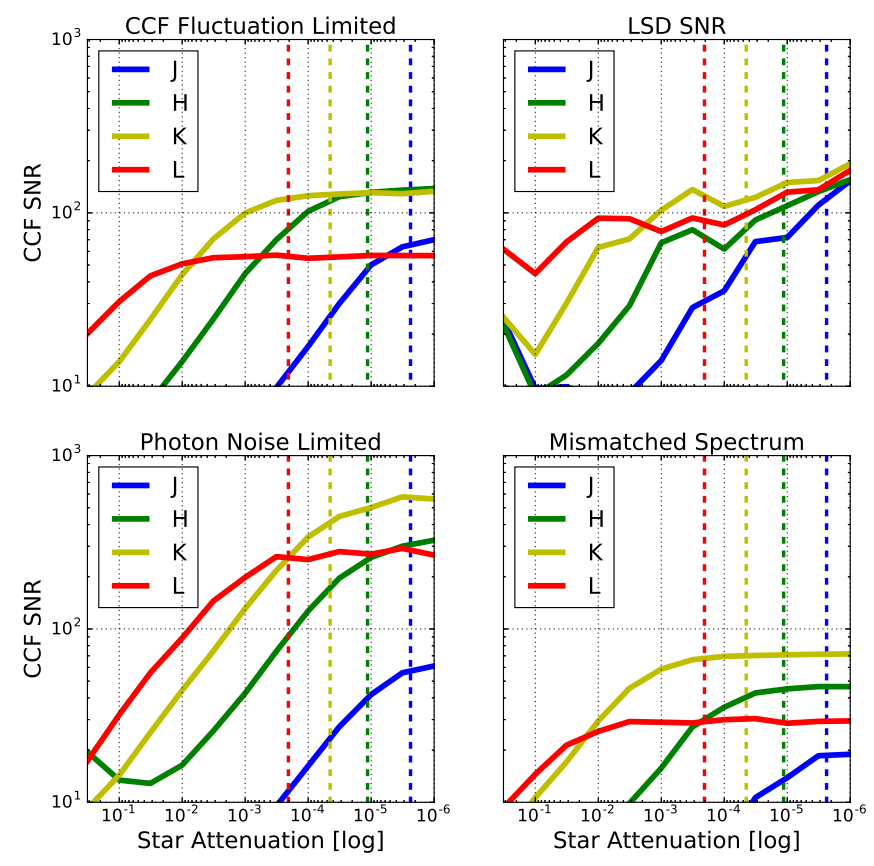

Figure 3. CCF SNR vs. star light attenuation level for HR 8799 e in four cases. Dashed lines indicate planet/star flux ratio for different bands.

\subsubsection{Optimal Band For Planet Detection}

Fig. 3 shows CCF SNRs at different levels of star light attenuation. For all cases of CCF SNR calculation, $K$ and $L$ bands are the optimal bands that deliver the highest CCF SNR. At low level of star light attenuation, $L$ band outperforms $K$ band. This is because the planet/star flux ratio is low in $L$ band and the photon noise form the leaked star light plays a relatively less important role. At high level of star light attenuation, $K$ band becomes the optimal band. The transition of performance between $L$ and $K$ band takes place at star light attenuation level between $\sim 10^{-2}$ and a few times $10^{-4}$ depending on different cases.

\subsubsection{Sensitivity Gain in HCI+HRS Observation}

Compared to ground-based HCI observations of HR 8799 e, HCI+HRS observation provides a significant gain in sensitivity. In $L$ band, detection significance is 5-10 [27]. Our simulation indicates that, at a level of star light attenuation of $10^{-3}$, CCF SNR in $L$ is between 20 (mismatch spectrum case) and 100 (photon noise limited case), a factor of $\sim 2-20$ gain in sensitivity with the help of HRS. This is because HRS serves as an additional filter for the planet signal. The gain due to HRS is limited by strong sky emission in $L$ band and/or other systematics. 
In other bands in which sensitivity is not limited by sky background but by planet/star flux ratio, we expect a HCI+HRS instrument provides higher gain in sensitivity. For example, planet/star flux ratio for HR $8799 \mathrm{e}$ is $\sim 4 \times 10^{-5}$, which may not be seen by a HCI instrument with star light attenuation level of $10^{-3}$. With a HCI+HRS instrument, the planet can be detected with a CCF SNR of 70-130 depending on different cases (Fig. $3)$.

\subsubsection{Doppler Imaging}

We simulate Doppler imaging observation for HR 8799 e. LSFs are calculated based on the LSD method with regularization that smooths LSFs at a certain SNR [26]. We analyze LSFs at different rotation phases with the MEM $[23,24]$. The MEM minimizes surface artifacts caused by insufficient SNR. The core equation in MEM is: $\mathrm{M}=\chi^{2}-\alpha \mathrm{S}$, where $\mathrm{M}$ is the quantity to minimize, $\chi^{2}$ quantifies the difference between input and inferred surface map, $\mathrm{S}$ is the entropy of surface map, and $\alpha$ controls the weight of $\mathrm{S}$ in $\mathrm{M}$. The MEM attempts to minimize $\chi^{2}$ and maximize $\mathrm{S}$, which is the smoothness of surface map. We follow the MEM detailed in MemSys5 manual [28], in which an automatic way of finding an optimal $\alpha$ is given depending on the SNR of the measurement.

LSF SNR is usually comparable with CCF SNR at high SNR regime [26]. We show in Fig. 3 that CCF SNR or LSF SNR is typically $>100$ for an hour observation in $K$ band. This allows for measurements of 8 phases within one rotation assuming that HR 8799 e has the same 8-hour rotation period as Beta Pic b [14]. Assuming 1 Jupiter radius, the rotation velocity of $\mathrm{HR} 8799 \mathrm{e}$ is $15 \mathrm{~km} \mathrm{~s}^{-1}$. For faster rotators such as Luhman $16 \mathrm{~B}$, shorter exposure time is needed in order to sample enough phases within one rotation. In such cases for fast rotators, LSF SNR would thus be reduced. In our simulation for Doppler imaging, we adopt a 8 by 4 surface grids along longitudinal and latitudinal direction. The choice of 8 grids along longitudinal direction is due to limited phase sampling given the exposure time and the rotation period of a planet. The choice of 4 grids along latitudinal direction is limited by the total number of constraints. There are $\sim 5-6$ pixels across each of the 8 LSFs, so the total number of constraints is $40-48$. We choose 4 grids along latitudinal direction to ensure the number of freedom (i.e., $8 \times 4=32$ ) is fewer than the total number of constraints.
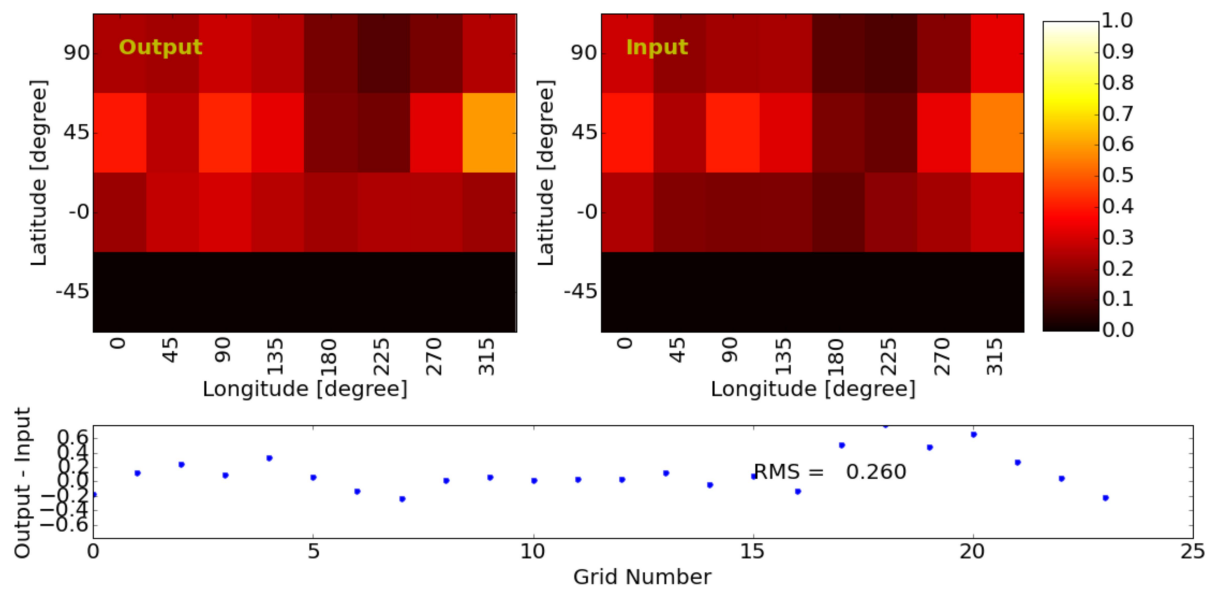

Figure 4. Simulating Doppler imaging observation for HR 8799 e. The inferred surface map has a $26.0 \%$ percentage error when compared to the input map. The main difference takes place for grids around equator where a portion of these grids is not visible because of the inclination of the planet $\left(i=28^{\circ}\right)$. Excluding the grids around equator, the percentage error is $13.8 \%$.

\section{SUMMARY}

HCI+HRS is a powerful technique for exoplanet detection and characterization. Compared to HCI, HRS serves as a spectral filter and brings additional sensitivity when the star light attenuation level is not high enough to detect an exoplanet. We develop a pipeline to simulate HCI+HRS observation and apply the pipeline to a benchmark planetary system, HR 8799 e. The simulation provides results to quantify the performance of 
HCI+HRS instruments and set system requirements from the science perspective. The requirements will thus inform design of an HCI+HRS instrument and its sub-systems, such as AO, chronograph, spectrograph and the FIU that links the coronagraphic system and the spectrograph system. We find that multi-band observation is important in fully characterizing the atmosphere of a planet. A good match between the planet spectrum and the model spectrum is critical in increasing the SNR of planet or molecular species detection.

\section{REFERENCES}

[1] Konopacky, Q. M., Barman, T. S., Macintosh, B. A., and Marois, C., "Detection of Carbon Monoxide and Water Absorption Lines in an Exoplanet Atmosphere," Science 339, 1398-1401 (Mar. 2013).

[2] Oppenheimer, B. R., Baranec, C., Beichman, C., Brenner, D., Burruss, R., Cady, E., Crepp, J. R., Dekany, R., Fergus, R., Hale, D., Hillenbrand, L., Hinkley, S., Hogg, D. W., King, D., Ligon, E. R., Lockhart, T., Nilsson, R., Parry, I. R., Pueyo, L., Rice, E., Roberts, J. E., Roberts, Jr., L. C., Shao, M., Sivaramakrishnan, A., Soummer, R., Truong, T., Vasisht, G., Veicht, A., Vescelus, F., Wallace, J. K., Zhai, C., and Zimmerman, N., "Reconnaissance of the HR 8799 Exosolar System. I. Near-infrared Spectroscopy," ApJ 768, 24 (May 2013).

[3] Bonnefoy, M., Zurlo, A., Baudino, J. L., Lucas, P., Mesa, D., Maire, A.-L., Vigan, A., Galicher, R., Homeier, D., Marocco, F., Gratton, R., Chauvin, G., Allard, F., Desidera, S., Kasper, M., Moutou, C., Lagrange, A.-M., Antichi, J., Baruffolo, A., Baudrand, J., Beuzit, J.-L., Boccaletti, A., Cantalloube, F., Carbillet, M., Charton, J., Claudi, R. U., Costille, A., Dohlen, K., Dominik, C., Fantinel, D., Feautrier, P., Feldt, M., Fusco, T., Gigan, P., Girard, J. H., Gluck, L., Gry, C., Henning, T., Janson, M., Langlois, M., Madec, F., Magnard, Y., Maurel, D., Mawet, D., Meyer, M. R., Milli, J., Moeller-Nilsson, O., Mouillet, D., Pavlov, A., Perret, D., Pujet, P., Quanz, S. P., Rochat, S., Rousset, G., Roux, A., Salasnich, B., Salter, G., Sauvage, J.-F., Schmid, H. M., Sevin, A., Soenke, C., Stadler, E., Turatto, M., Udry, S., Vakili, F., Wahhaj, Z., and Wildi, F., "First light of the VLT planet finder SPHERE. IV. Physical and chemical properties of the planets around HR8799," A\&\&A 587, A58 (Mar. 2016).

[4] Rajan, A., Barman, T., Soummer, R., Brendan Hagan, J., Patience, J., Pueyo, L., Choquet, É., Konopacky, Q., Macintosh, B., and Marois, C., "Characterizing the Atmospheres of the HR8799 Planets with HST/WFC3," ApJL 809, L33 (Aug. 2015).

[5] Snellen, I., de Kok, R., Birkby, J. L., Brandl, B., Brogi, M., Keller, C., Kenworthy, M., Schwarz, H., and Stuik, R., "Combining high-dispersion spectroscopy with high contrast imaging: Probing rocky planets around our nearest neighbors," A\&A 576, A59 (Apr. 2015).

[6] Snellen, I. A. G., de Kok, R. J., de Mooij, E. J. W., and Albrecht, S., "The orbital motion, absolute mass and high-altitude winds of exoplanet HD209458b," Nature 465, 1049-1051 (June 2010).

[7] Birkby, J. L., de Kok, R. J., Brogi, M., de Mooij, E. J. W., Schwarz, H., Albrecht, S., and Snellen, I. A. G., "Detection of water absorption in the day side atmosphere of HD $189733 \mathrm{~b}$ using ground-based high-resolution spectroscopy at $3.2 \mu \mathrm{m}$," MNRAS 436, L35-L39 (Nov. 2013).

[8] de Kok, R. J., Brogi, M., Snellen, I. A. G., Birkby, J., Albrecht, S., and de Mooij, E. J. W., "Detection of carbon monoxide in the high-resolution day-side spectrum of the exoplanet HD 189733b," A\& A 554, A82 (June 2013).

[9] Kempton, E. M.-R., Perna, R., and Heng, K., "High Resolution Transmission Spectroscopy as a Diagnostic for Jovian Exoplanet Atmospheres: Constraints from Theoretical Models," ApJ 795, 24 (Nov. 2014).

[10] Brogi, M., Snellen, I. A. G., de Kok, R. J., Albrecht, S., Birkby, J., and de Mooij, E. J. W., "The signature of orbital motion from the dayside of the planet $\tau$ Boötis b," Nature 486, 502-504 (June 2012).

[11] Brogi, M., Snellen, I. A. G., de Kok, R. J., Albrecht, S., Birkby, J. L., and de Mooij, E. J. W., "Detection of Molecular Absorption in the Dayside of Exoplanet 51 Pegasi b?," ApJ 767, 27 (Apr. 2013).

[12] Brogi, M., de Kok, R. J., Birkby, J. L., Schwarz, H., and Snellen, I. A. G., "Carbon monoxide and water vapor in the atmosphere of the non-transiting exoplanet HD 179949 b," A 6 A 565, A124 (May 2014).

[13] Lockwood, A. C., Johnson, J. A., Bender, C. F., Carr, J. S., Barman, T., Richert, A. J. W., and Blake, G. A., "Near-IR Direct Detection of Water Vapor in Tau Boötis b," ApJL 783, L29 (Mar. 2014).

[14] Snellen, I. A. G., Brandl, B. R., de Kok, R. J., Brogi, M., Birkby, J., and Schwarz, H., "Fast spin of the young extrasolar planet $\beta$ Pictoris b," Nature 509, 63-65 (May 2014). 
[15] Crossfield, I. J. M., Biller, B., Schlieder, J. E., Deacon, N. R., Bonnefoy, M., Homeier, D., Allard, F., Buenzli, E., Henning, T., Brandner, W., Goldman, B., and Kopytova, T., "A global cloud map of the nearest known brown dwarf," Nature 505, 654-656 (Jan. 2014).

[16] Baraffe, I., Homeier, D., Allard, F., and Chabrier, G., "New evolutionary models for pre-main sequence and main sequence low-mass stars down to the hydrogen-burning limit," $A \mathscr{E} A \mathbf{5 7 7}$, A42 (May 2015).

[17] Benneke, B., "Strict Upper Limits on the Carbon-to-Oxygen Ratios of Eight Hot Jupiters from SelfConsistent Atmospheric Retrieval," ArXiv e-prints (Apr. 2015).

[18] Hu, R., Ehlmann, B. L., and Seager, S., "Theoretical Spectra of Terrestrial Exoplanet Surfaces," ApJ 752, 7 (June 2012).

[19] Hu, R., Seager, S., and Bains, W., "Photochemistry in Terrestrial Exoplanet Atmospheres. I. Photochemistry Model and Benchmark Cases," ApJ 761, 166 (Dec. 2012).

[20] Hu, R., Seager, S., and Bains, W., "Photochemistry in Terrestrial Exoplanet Atmospheres. II. $\mathrm{H}_{2} \mathrm{~S}$ and $\mathrm{SO}_{2}$ Photochemistry in Anoxic Atmospheres," ApJ 769, 6 (May 2013).

[21] Hu, R. and Seager, S., "Photochemistry in Terrestrial Exoplanet Atmospheres. III. Photochemistry and Thermochemistry in Thick Atmospheres on Super Earths and Mini Neptunes," ApJ 784, 63 (Mar. 2014).

[22] Lord, S. D., "A New Software Tool for Computing Earth's Atmospheric Transmission of Near- and FarInfrared Radiation," NASA Technical Memorandum, 103957 (Mar. 1992).

[23] Gull, S. F. and Daniell, G. J., "Image reconstruction from incomplete and noisy data," Nature 272, 686-690 (Apr. 1978).

[24] Gull, S. and Skilling, J., "Maximum entropy method in image processing," Communications, Radar and Signal Processing, IEE Proceedings F 131, 646-659 (October 1984).

[25] Bonnefoy, M., Zurlo, A., Baudino, J. L., Lucas, P., Mesa, D., Maire, A.-L., Vigan, A., Galicher, R., Homeier, D., Marocco, F., Gratton, R., Chauvin, G., Allard, F., Desidera, S., Kasper, M., Moutou, C., Lagrange, A.-M., Baruffolo, A., Baudrand, J., Beuzit, J.-L., Boccaletti, A., Cantalloube, F., Carbillet, M., Charton, J., Claudi, R. U., Costille, A., Dohlen, K., Dominik, C., Fantinel, D., Feautrier, P., Feldt, M., Fusco, T., Gigan, P., Girard, J. H., Gluck, L., Gry, C., Henning, T., Janson, M., Langlois, M., Madec, F., Magnard, Y., Maurel, D., Mawet, D., Meyer, M. R., Milli, J., Moeller-Nilsson, O., Mouillet, D., Pavlov, A., Perret, D., Pujet, P., Quanz, S. P., Rochat, S., Rousset, G., Roux, A., Salasnich, B., Salter, G., Sauvage, J.-F., Schmid, H. M., Sevin, A., Soenke, C., Stadler, E., Turatto, M., Udry, S., Vakili, F., Wahhaj, Z., and Wildi, F., "First light of the VLT planet finder SPHERE. IV. Physical and chemical properties of the planets around HR8799," ArXiv e-prints (Nov. 2015).

[26] Kochukhov, O., Makaganiuk, V., and Piskunov, N., "Least-squares deconvolution of the stellar intensity and polarization spectra," A $\mathscr{G} A \mathbf{5 2 4}$, A5 (Dec. 2010).

[27] Currie, T., Burrows, A., Girard, J. H., Cloutier, R., Fukagawa, M., Sorahana, S., Kuchner, M., Kenyon, S. J., Madhusudhan, N., Itoh, Y., Jayawardhana, R., Matsumura, S., and Pyo, T.-S., "Deep Thermal Infrared Imaging of HR 8799 bcde: New Atmospheric Constraints and Limits on a Fifth Planet," ApJ 795, 133 (Nov. 2014).

[28] Gull, S. and Skilling, J., [Quantified Maximum Entropy MemSys5 User's Manual: This Manual Describes the MemSys5 Package of Fortran 77 Subroutines Designed to Find and Use Maximum Entropy Reconstructions of Images, Power Spectra, and Other Such Addictive Distributions from Arbitrary Sets of Data], Maximum Entropy Data Consultants (1991). 